BULL. AUSTRAL. MATH. SOC.

VOL. $12(1975), 149-153$.

\title{
Nilpotent measures on compact semigroups
}

\section{H.L. Chow}

Let $S$ be a compact semigroup and $P(S)$ the set of probability measures on $S$. Suppose $P(S)$ has zero $\theta$ and define a measure $\mu \in P(S)$ nilpotent if $\mu^{n} \rightarrow \theta$. It is shown that any measure with support containing that of $\theta$ is nilpotent, and the set of nilpotent measures is convex and dense in $P(S)$. A measure $\mu$ is called mean-nilpotent if $\left(\mu+\mu^{2}+\ldots+\mu^{n}\right) / n \rightarrow \theta$, and can be characterized in terms of its support.

Throughout this paper $S$ is a compact topological semigroup such that its minimal ideal $K(S)$ is a (compact) group. As is well-known, the set $P(S)$ of probability measures on $S$ is a compact semigroup under convolution and the weak topology, [3]. For $\mu, \nu \in P(S)$, we then have [3, Lemma 2.1],

$$
\text { supp } \mu \nu=\text { supp } \mu \text { supp } \nu,
$$

where supph is the support of $\mu$, and so on. It follows that the (normalized) Haar measure $\theta$ of $K(S)$ is the zero of $P(S)$. Then a measure $\mu \in P(S)$ is said to be nilpotent if $\mu^{n} \rightarrow \theta$ as $n \rightarrow \infty$, and we denote by $N$ the set of nilpotent elements in $P(S)$. In [2], the case when $K(S)$ is a singleton has been considered; we established a characterization of nilpotent measures in terms of their supports, and examined the set $N$. It is the purpose of this note to obtain some possible extensions of those results to the general situation.

Received 12 November 1974. 
For a subset $A$ of $S$ or $P(S)$, let $S(A)$ denote the closed semigroup generated by $A$ and $K(S(A))$ its minimal ideal. In case $A=\{x\}$, we write $S(x)$ for $S(A)$. Recall that $K(S(x))$ is a group containing exactly the cluster points of the sequence $\left\{x^{n}\right\}$., (see $[4$, Theorem 3.1.1]). Now if $A \subset P(S)$, we write $\operatorname{supp} A=\overline{U \text { supp } \mu}$, where the bar denotes closure; it follows that $\operatorname{supp} S(A)=S(\operatorname{supp} A)$, by the same argument given in the proof of [2, Lemma 3]. Moreover, the minimal ideal of $\operatorname{supp} S(A)$ is $K(\operatorname{supp} S(A))=\operatorname{supp} K(S(A)) ;$ see $[1$, Theorem 5$]$.

Let $N_{0}=\{\mu \in P(S):$ supp $\mu \supset K(S)\}$, which is easily seen to be an ideal of $P(S)$.

THEOREM 1. $N_{0} \subset N$; that is, any measure with support containing $K(S)$ is nilpotent.

Proof. Take $\mu \in N_{0}$ and let $\tau$ be the identity of the group $K(S(\mu))$. Then $\operatorname{supp} \tau \subset \operatorname{supp} K(S(\mu))=K(\operatorname{supp} S(\mu))=K(S(\operatorname{supp} \mu))=K(S)$. Hence supp $\mu \tau=$ suppusupp $\tau \supset K(S)_{\text {supp } \tau=K(S)}$. On the other hand, supp $\mu \tau \subset K(S)$ since $\mu \tau \in K(S(\mu))$. Consequently supp $\mu \tau=K(S)$. Since $K(S(\mu))$ is a group, there exists $\nu \in K(S(\mu))$ such that $(\mu \tau) \nu=\tau$. It follows, therefore, that suppt $=$ supputsuppv $=K(S)_{\text {supp }}=K(S)$, since suppv $\subset K(S)$. By virtue of Theorem 1 of [6], we see that $\tau$ is the Haar measure of $K(S)$; that is, $\tau=\theta$, whence $K(S(\mu))=\{\theta\}$. This means that the sequence $\left\{\mu^{n}\right\}$ has only one cluster point $\theta$ and so $\mu^{n} \rightarrow \theta$, that is $\mu \in N$, completing the proof.

REMARK. Equality need not hold in the theorem above. For instance let $S$ be the multiplicative semigroup of real numbers in the closed unit interval with the usual topology. If $\delta\left(\frac{1}{2}\right)$ denotes the Dirac measure at $\frac{1}{2} \in S$, clearly $\delta\left(\frac{1}{2}\right) \in N \backslash N_{0}$.

COROLLARY 2. Let $\mu \in P(S)$ and supp $\mu^{n} \supset K(S)$ for some positive integer $n$; then $\mu \in N$.

Proof. In view of the fact that $\mu^{n} \in N_{0} \subset N$, we see $\mu \in N$ by Lemma 2.1 .4 of [4]. 
THEOREM 3. The set $N_{0}$ is convex and everywhere dense, and $\operatorname{supp} N_{0}=S$

Proof. The argument parallels that in [2]. First, taking $\mu, \nu \in N_{0}$, we let $\tau=t \mu+(I-t) \nu$ for $0<t<1$. Then supp $\tau=\operatorname{supp} \mu \cup \operatorname{supp} \nu \supset K(S)$ implies $\tau \in N_{0}$; that is, $N_{0}$ is convex. To see that $N_{0}$ is everywhere dense, let $\lambda \in P(S)$ and consider $\lambda_{n}=\frac{1}{n} \theta+\frac{n-I}{n} \lambda$ for positive integers $n$. It is obvious that $\lambda_{n} \in N_{0}$ and $\lambda_{n} \rightarrow \lambda$ as $n \rightarrow \infty$; that is, $N_{0}$ is dense in $P(S)$. Finally, for any $x \in S$, let $\omega=\frac{1}{2}(\theta+\delta(x))$. Since $x \in$ supp $\omega$ and $\omega \in N_{0}$, the result is immediate.

THEOREM 4. The set $N$ is connected and everywhere dense, and $\operatorname{suppN}=S$.

Proof. By Theorem 3, $N_{0}$ is convex and so connected. Moreover, $N_{0}$ is everywhere dense and $N \supset N_{0}$, whence $N$ is connected. The rest is clear.

Note that $N$ is convex when $K(S)$ is a singleton, (see [2, Theorem 12]). But, in the present case, we can only establish the connectedness of $N$.

Given $\mu \in P(S)$, it is known that the sequence $\left(\frac{\mu+\mu^{2}+\ldots+\mu^{n}}{n}\right)$ must converge to an idempotent measure $L(\mu)$ with $\operatorname{supp} L(\mu)=K(S(\operatorname{supp} \mu))$, [5]. The measure $\mu$ is termed mean-nilpotent if $L(\mu)=\theta$. Let $M$ denote the set of mean-nilpotent measures on $S$; it is evident that $M \supset N$; that is, a nilpotent measure is mean-nilpotent. That the converse does not hold may be seen from the example below. But first we characterize mean-nilpotency in the next theorem.

THEOREM 5. Let $\mu \in P(S)$. Then $\mu \in M$ if and only if $S(\operatorname{supp} \mu) \supset K(S)$.

Proof. To prove the "if" part, we note that $S($ supp $\mu) \supset K(S)$ implies $K(S(\operatorname{supp} \mu))=K(S)$, whence $\operatorname{supp} L(\mu)=K(S)$. Accordingly, $L(\mu)=\theta$; that is, $\mu \in M$. Conversely, since 


$$
S(\operatorname{supp} \mu) \supset K(S(\operatorname{supp} \mu))=\operatorname{supp} L(\mu)=\operatorname{supp} \theta=K(S) \text {, }
$$

the "only if" part follows easily.

COROLLARY 6. If $\mu^{n} \in M$ for some $n$, then $\mu \in M$.

Proof. Because $S($ supp $\mu) \supset S\left(\right.$ supp $\left.^{n}\right)$, we apply the previous theorem to obtain the result.

COROLLARY 7. The set $M$ is convex and everywhere dense, and $\operatorname{supp} M=S$.

Proof. We need only to show that $M$ is convex. Take $\mu, \nu \in M$ and let $\tau=t \mu+(1-t) \nu$ for $0<t<1$. Since

$$
\text { suppt }=\text { supp } u \text { supp } \supset \supset \text { supp } \mu \text {, }
$$

it follows that $S(\operatorname{supp} \tau) \supset S(\operatorname{supp} \mu) \supset K(S)$. This together with Theorem 5 gives $\tau \in M$.

EXAMPLE 8. A mean-nilpotent measure is not nilpotent. Take the group $S=\{a, e\}, e$ being the identity, and let $\mu=\delta(a)$. Then $\mu \in M$ since $S($ supp $\mu)=S(a)=S=K(S)$, but $\mu$ is clearly not nilpotent.

When the group $K(S)$ is a singleton, it can be shown that a measure in $P(S)$ is nilpotent if and only if mean-nilpotent, [2, Theorem 14], so that nilpotency of measures is dependent of supports only. The author has been unable to prove whether this carries over to the general case. That is, given $\mu, \nu \in P(S)$ with supp $\mu=$ supp $\nu$, if $\mu \in M$, is it true that $\nu \in N$ also?

\section{References}

[1] H.L. Chow, "On supports of semigroups of measures", Proc. Edinburgh Math. Soc. (2) 19 (1974), 31-33.

[2] H.L. Chow, "Quasi-nilpotent sets in semigroups", Proc. Amer. Math. Soc. (to appear).

[3] Irving Glicksberg, "Convolution semigroups of measures", Pacific $J$. Math. 9 (1959), 51-67. 
[4] A.B. Paalman-de Miranda, Topological semigroups, 2nd ed. (Mathematical Centre Tracts, 11. Mathematisch Centrum, Amsterdam, 1970).

[5] Stefan Schwarz, "Convolution semigroup of measures on compact noncommutative semigroups", Czechoslovak Math. J. 14 (89) (1964), 95-115.

[6] J.G. Wendel, "Haar measure and the semigroup of measures on a compact group", Proc. Amer. Math. Soc. 5 (1954), 923-929.

Department of Mathematics,

Chung Chi College,

The Chinese University of Hong Kong,

Hong Kong. 